PROCEEDINGS OF THE

AMERICAN MATHEMATICAL SOCIETY

Volume 125, Number 12, December 1997, Pages 3581-3592

S 0002-9939(97)04035-5

\title{
ON A CONJECTURE OF RIDGE
}

\author{
TIN-YAU TAM \\ (Communicated by Palle E. T. Jorgensen)
}

\begin{abstract}
The conjecture of Ridge on the numerical range of a shift of periodic weights is resolved in the affirmative, i.e., if the weights are nonzero, the numerical range of the corresponding shift is an open disc centered at the origin. The radius of the disc can be expressed as the Perron root of a nonnegative irreducible symmetric matrix. Some related results are obtained.
\end{abstract}

\section{INTRODUCTION}

A unilateral weighted shift on $\ell_{+}^{2}$ is a bounded linear operator $S$ defined by $S e_{n}=s_{n} e_{n+1}, n=1,2, \ldots$, where $\left\{e_{n}\right\}$ is an orthonormal basis, and $\left\{s_{n}\right\}$ is a sequence of complex numbers. The numerical range of $S$, denoted by $W(S)$, is the subset of the complex plane: $W(S)=\left\{(S x, x): x \in \ell_{+}^{2},\|x\|=1\right\}$. The weighted bilateral shift $B$ on $\ell^{2}$ and its numerical range are defined analogously.

We list some well-known facts about weighted shifts [H], [R2], [S]:

(1) $S=U D$ where $U$ is the unilateral shift and $D$ is the diagonal operator defined as $D e_{n}=s_{n} e_{n}$ for all positive integers $n$. The shift $S$ is a bounded operator if and only if $\left\{s_{n}\right\}$ is a bounded sequence. Since $U$ is an isometry, $\|S\|=\|D\|=\sup \left|s_{n}\right|$.

(2) $S$ is unitarily equivalent to a shift with weights $t_{n}$ where $\left|t_{n}\right|=\left|s_{n}\right|$ for all $n$. In particular, $S$ is unitarily equivalent to $c S$ whenever $|c|=1$. Hence the spectrum and the numerical range of $S$ both have circular symmetry about 0 . Since the numerical range is always convex (Toeplitz-Hausdorff Theorem) [H, Problem 166], $W(S)$ is a circular disc centered at the origin (one may see that the origin is contained in $W(S)$ as $0=\left(S e_{n}, e_{n}\right)$ ), either open or closed.

The above properties are also true for weighted bilateral shifts.

Due to (2), in the rest of the dicussion we will assume that $s_{n} \geq 0$.

We denote by $\mathcal{B}(\mathcal{H})$ the Banach algebra of bounded linear operators on the Hilbert space $\mathcal{H}$. For $A \in \mathcal{B}(\mathcal{H}), A_{+}=\left(A+A^{*}\right) / 2$ and $A_{-}=\left(A-A^{*}\right) / 2$ are the Hermitian and skew Hermitian parts of $A$, respectively. The projection of $W(A)$ onto the real line, $\operatorname{Re} W(A)$, is $W\left(A_{+}\right)$. The numerical radius of $A$ is defined as $w(A)=\sup \{|z|: z \in W(A)\}$. Since we will use the following result repeatedly, we would like to state it explicitly.

Received by the editors February 22, 1996, and, in revised form, July 3, 1996.

1991 Mathematics Subject Classification. Primary 47A12, 47B20.

Key words and phrases. Weighted shift, numerical range.

Some results of the paper have been presented in the Third Matrix Theory Mini-Conference in Hong Kong, June, 1995. 
Proposition $1([\mathrm{H}]$, Problem 168). Let $A \in \mathcal{B}(\mathcal{H})$. If $\lambda \in W(A)$ and $|\lambda|=\|A\|$, then $\lambda$ is an eigenvalue of $A$.

In [R2, p.107] Ridge asserted that if $s_{n} \leq K$ for all $n$, and $s_{n} \rightarrow K$ then $w(S)=K$. The main reason is that the inequalities $\rho(A) \leq w(A) \leq\|A\|$ are true for any $A \in \mathcal{B}(\mathcal{H})$, where $\rho(A)$ is the spectral radius of $A$. For a weighted shift $S$, $\rho(S)=\lim _{n} \sup _{k}\left(s_{k+1} \ldots s_{k+n}\right)^{1 / n}$ [R1]. Hence if $s_{n} \leq K$ for all $n$, and $s_{n} \rightarrow K$, then $\rho(A)=\|A\|=K$. This implies that $w(S)=K$.

We would like to prove the same result without using Ridge's expression of the spectral radius of $S$ and show that $W(S)$ is an open disc under the same condition, by using Proposition 1. In order to avoid triviality, we assume that $K>0$. We will also deal with the bilateral case.

Theorem 1. (i) Let $S \in B\left(\ell_{+}^{2}\right)$ be a unilateral weighted shift with weights $\left\{s_{n}\right\}$. If $s_{n} \leq K$ for all positive integers $n$, and $\lim _{n \rightarrow \infty} s_{n}=K>0$, then $W(S)$ is the open disc centered at the origin with radius $K$.

(ii) Let $S \in B\left(\ell^{2}\right)$ be a bilateral weighted shift with weights $\left\{s_{n}\right\}$. If $s_{n} \leq K$ for all integers $n$, and $\lim _{n \rightarrow \infty} s_{n}=K>0$ or $\lim _{n \rightarrow \infty} s_{-n}=K>0$, then $W(B)$ is the open disc centered at the origin with radius $K$.

Proof. (i) Let $x=(0,0, \ldots, 0,1 / \sqrt{r}, 1 / \sqrt{r}, \ldots 1 / \sqrt{r}, 0,0,0, \ldots)$ where we have $r$ copies of zero and then $r$ copies of $1 / \sqrt{r}$ and zeros afterward. Then $x \in \ell_{+}^{2}$ with $\|x\|=1$ and $(S x, x)=\left(s_{r+1}+s_{2}+\cdots+s_{2 r-1}\right) / r$. As $s_{n} \rightarrow K$, we have $(S x, x) \rightarrow K$ as $r \rightarrow \infty$, i.e., $w(S) \geq K$. But $w(S) \leq\|S\|=K$, so we have $w(S)=K$.

Since $s_{n} \rightarrow K>0$, there are finitely many zeros (maybe none) among $s_{n}$ 's. Let $s_{r}=0$ be the last zero. Then $S=N \oplus M$ where $N$ is a nilpotent operator on an $r$ dimensional space and $M$ is a unilateral weighted shift on $l_{+}^{2}$ with positive weights. The point spectrum of $M$ is empty [H, Problem 78]. So the point spectrum of $S$ is either empty or the singleton set $\{0\}$. Hence $K$ cannot be an eigenvalue of $S$ and then $W(S)$ is open by Proposition 1.

(ii) The same argument in (i) yields that $w(B)=K$. We are going to show that $W(B)$ is open. Let $V$ be the usual bilateral shift, i.e., weights are all 1 . The operator $V$ has no eigenvalue [H, Problem 68]. Then by Proposition 1, $W(V)$ is the open unit disc. Recall that $W(B)=\left\{\sum_{n} s_{n} x_{n} x_{n+1}: \sum_{n}\left|x_{n}\right|^{2}=1\right\}$. If $W(B)$ were closed, then there would be an $x \in \ell^{2}$ with $\|x\|=1$ such that $K=\sum_{n} s_{n} x_{n} x_{n+1}$. The vector $x$ can be selected such that the coordinates of $x$ are all real and nonnegative in view of the triangle inequality. Then $K(V x, x)=$ $\sum_{n} K x_{n} x_{n+1} \geq \sum_{n} s_{n} x_{n} x_{n+1}=K$ as $s_{n} \leq K$ for all $n$. It would imply that $1 \in W(V)$ but this is impossible, as $W(V)$ is an open disc.

We remark that the above argument is also applicable to (i) as $W(U)$ is the open unit disc.

An operator $A \in \mathcal{B}(\mathcal{H})$ is hyponormal if $\|A x\| \geq\left\|A^{*} x\right\|$ for all $x \in \mathcal{H}$, or equivalently, $A^{*} A-A A^{*} \geq 0$.

Corollary 1. The numerical range of a hyponormal weighted shift with weights $\left\{s_{n}\right\}$ is the open disc centered at the origin with the operator norm as the radius.

Proof. A weighted shift is hyponormal if and only if its weight sequence $\left\{s_{n}\right\}$ is increasing $[\mathrm{C}, \mathrm{p} .55]$ with operator norm $\sup s_{n}$. In other words, $\left\{s_{n}\right\}$ satisfies the conditions in Theorem 1. 
Example 1. One can easily construct a unilateral weighted shift $S$ with open $W(S)$ without satisfying the conditions in Theorem 1 (i), e.g., $S=N \oplus U$ where

$$
\left(\begin{array}{ll}
0 & 0 \\
\alpha & 0
\end{array}\right), \quad 1<\alpha<2
$$

and $U$ is the unilateral shift. But here is another example [H, Problem 81] which also fits. (It implies the existence of a countable set of operators, each with spectrum $\{0\}$, whose direct sum has spectral radius 1 . See [Ch] for related results.) Let $S$ be the unilateral weighted shift with weights $(1,0,1,1,0,1,1,1,0,1,1,1,1,0, \ldots)$, i.e., $S$ is the direct sum of the finite dimensional nilpotent operators

$$
\left(\begin{array}{ll}
0 & 0 \\
1 & 0
\end{array}\right), \quad\left(\begin{array}{lll}
0 & 0 & 0 \\
1 & 0 & 0 \\
0 & 1 & 0
\end{array}\right), \quad\left(\begin{array}{llll}
0 & 0 & 0 & 0 \\
1 & 0 & 0 & 0 \\
0 & 1 & 0 & 0 \\
0 & 0 & 1 & 0
\end{array}\right), \quad \ldots
$$

If we denote the above nilpotent operators by $N_{n}, n \geq 2$, then $S=\oplus N_{n}$ and $W(S)$ is the open disc centered at the origin with radius $|\cos [n \pi /(n+1)]|$ (see Proposition 2 or [MS, Lemma 3]). So $W\left(N_{n}\right) \subset W\left(N_{n+1}\right)$ for all positive integers $n$. The numerical radius of $S$ is 1 as $w\left(N_{n}\right)=|\cos [n \pi /(n+1)]| \rightarrow 1$ when $n \rightarrow 1$. If 1 were in $W(S)$, it would be an eigenvalue of $S$ by Proposition 1. But the point spectrum of $S$ is just the union of the point spectra of $N_{n}$, which is empty.

Remark 1. In view of the above example, one may try to replace the condition $s_{n} \rightarrow K$ by $\lim \sup _{n} s_{n}=K$. But the statements of Theorem 1 are no longer valid. Define $s_{n}=1$ for even $n$ and $s_{n}=0$ for odd $n$. Then $S(B)$ is just a direct sum of countably many copies of the matrix

$$
\left(\begin{array}{ll}
0 & 0 \\
1 & 0
\end{array}\right)
$$

and hence $W(S)(W(B))$ is the closed disc centered at the origin with radius $1 / 2$.

If $s_{n}>K$ for some $n$, then $w(S)$ and $w(B)$ can be greater than $K$ and $W(S)$ can be closed. See Proposition 2 and 3.

In order to describe $W(S)$ completely for a general weighted shift $S$, we have to find the numerical radius of $S$ and determine whether $W(S)$ is closed or not.

We have the following result.

Theorem 2. Let $A \in \mathcal{B}(\mathcal{H})$. Then $w(A) \in W(A)$ if and only if $w(A)$ is an eigenvalue of $A_{+}$.

Proof. If $w(A)$ is an eigenvalue of $A_{+}$, i.e., $A_{+} x=w(A) x$ for some unit vector $x$, then $(A x, x)=\left(A_{+} x, x\right)+\left(A_{-} x, x\right)=w(A)+\left(A_{-} x, x\right)$. Since $\left(A_{-} x, x\right)$ is purely imaginary and $w(A) \geq 0$ is the numerical radius of $A$, we have $\left(A_{-} x, x\right)=0$. So $w(A)=(A x, x) \in W(A)$.

Conversely, if $w(A) \in W(A)$, then $w(A) \in W\left(A_{+}\right)$as $w(A) \geq 0$. But $\left\|A_{+}\right\|=$ $w\left(A_{+}\right)$for the operator $A_{+}$is Hermitian, and hence $w(A)$ is an eigenvalue of $A_{+}$ by Proposition 1 .

Corollary 2. Let $A \in \mathcal{B}(\mathcal{H})$ such that $W(A)$ is a disc centered at the origin, either open or closed. Then $W(A)$ is closed if and only if $w(A)$ is an eigenvalue of $A_{+}$.

Proof. The assumptions on $A$ imply that $W(A)$ is closed if and only if $w(A) \in$ $W(A)$. Then the result follows from Theorem 2 . 
Example 2. The condition that $W(A)$ is either open or closed is necessary. The operator $A=1 \oplus U$ where $U$ is the unilateral shift has $W(A)=\operatorname{conv}\{1, W(U)\}$. This is the open unit disc joining with the point 1 .

Corollary 3. Let $S \in B\left(\ell_{+}^{2}\right)\left(B \in B\left(\ell^{2}\right)\right)$ be a unilateral (bilateral) weighted shift. Then $W(S)(W(B))$ is closed if and only if $w(S)(w(B))$ is an eigenvalue of $S_{+}$ $\left(B_{+}\right)$.

However, as noticed by Shields [S, p.73], there does not seem to be a simple formula for the numerical radius of a general weighted shift in view of the following example.

Proposition 2. Let $S_{k} \in B\left(\ell_{+}^{2}\right)$ be defined as $S_{k} e_{1}=k e_{2}$ and $S_{k} e_{n}=e_{n+1}$ for $n>1$, where $0 \leq k$.

(i) If $\sqrt{2}<k$, then $W\left(S_{k}\right)$ is the closed disc with radius $\frac{k^{2}}{2\left(k^{2}-1\right)^{1 / 2}}$.

(ii) If $0 \leq k \leq \sqrt{2}$, then $W\left(S_{k}\right)$ is the open unit disc.

Proof. (i) See [BS, p.1053-1054] for the nice calculation of Shields. In the proof $W\left(M_{z}\right)$ should be the open unit disc instead of the closed unit disc [BS, p.1054].

(ii) Since the function $w: \mathcal{B}(\mathcal{H}) \rightarrow \mathbb{R}$ is continuous with respect to the uniform (norm) topology [H, Problem 175], we have $w\left(S_{\sqrt{2}}\right)=\lim _{k \rightarrow \sqrt{2}} \frac{k^{2}}{2\left(k^{2}-1\right)^{1 / 2}}=1$.

As $W\left(S_{k}\right) \subset W\left(S_{k}^{\prime}\right)$ if $k \leq k^{\prime}$, we have $w\left(S_{k}\right)=1$ when $0 \leq k \leq \sqrt{2}$.

When $0 \leq k \leq 1$, we simply apply Theorem 1 (i) to conclude that $W\left(S_{k}\right)$ is the open unit disc.

When $1<k \leq \sqrt{2}$, it is sufficient to show that 1 is not an eigenvalue of $\left(S_{k}\right)_{+}$ in view of Corollary 3. Suppose that 1 were an eigenvalue of $S_{k}$, i.e., there would exist $x=\sum_{n} x_{n} e_{n} \in \ell_{+}^{2}$ such that $\left(S_{k}\right)_{+} x=x$, i.e.,

$$
\begin{aligned}
k x_{2} & =2 x_{1}, \\
k x_{1}+x_{3} & =2 x_{2}, \\
x_{n}+x_{n+2} & =2 x_{n+1}, \quad n=2,3, \ldots
\end{aligned}
$$

Solving the equations, we have

$$
\begin{aligned}
x_{2} & =\frac{2}{k} x_{1}, \\
x_{3} & =2 x_{2}-k x_{1}=\frac{4-k^{2}}{k} x_{1}, \\
x_{n+2} & =2 x_{n+1}-x_{n}, \quad n=2,3, \ldots
\end{aligned}
$$

If $1<k \leq \sqrt{2}$, we have $x_{2}>x_{1}$ and $x_{3} \geq \frac{2}{k} x_{1}=x_{2}$. Then $x_{n+2}=2 x_{n+1}-x_{n}=$ $x_{n+1}+\left(x_{n+1}-x_{n}\right) \geq x_{n+1}$ by induction, i.e., $\left\{x_{n}\right\}$ is a nondecreasing sequence. This would imply $x \notin \ell_{+}^{2}$. So 1 is not an eigenvalue of $\left(S_{k}\right)_{+}$for $1<k \leq \sqrt{2}$.

Why is $\sqrt{2}$ the cutting point, i.e., why does the numerical radius of $S_{k}$ fail to increase until $k$ hits $\sqrt{2}$ ? Perhaps the following observation together with Ritz's theorem [L, p.260] offers some explanation. Let $U$ be the unilateral shift and let $P_{n}$ be the orthogonal projection of $\ell_{+}^{2}$ onto the span of $\left\{e_{1}, \ldots, e_{n}\right\}$. Then the finite 
dimensional operator $U_{n}=P_{n} U P_{n}$ has the matrix representation of size $n$ :

$$
\left(\begin{array}{ccccc}
0 & 0 & 0 & \ldots & 0 \\
1 & 0 & 0 & \ldots & 0 \\
0 & 1 & 0 & \ldots & 0 \\
& \ldots & \ldots & & \\
0 & 0 & \ldots & 1 & 0
\end{array}\right) .
$$

Since $W(U)$ is the open unit disc, we have $w(U)=w\left(U_{+}\right)$. According to Ritz's theorem [L, p.260], the largest eigenvalue of $\left(U_{r}\right)_{+}$tends to $w\left(U_{+}\right)$as $r \rightarrow \infty$. Let $f_{r}(\lambda)$ be the characteristic polynomial of $\left(U_{r}\right)_{+}$. By Laplace expansion along the $(n+1)^{s t}$ row of $\left(U_{2 n+1}\right)_{+}$, we have

$$
\begin{aligned}
f_{2 n+1}(\lambda) & =\operatorname{det}\left(\lambda I-\left(U_{2 n+1}\right)_{+}\right) \\
& =\lambda f_{n}^{2}(\lambda)-2 f_{n-1} f_{n}(\lambda) \\
& =\left[\lambda f_{n}(\lambda)-2 f_{n-1}(\lambda)\right] f_{n}(\lambda) .
\end{aligned}
$$

The expression $\left[\lambda f_{n}(\lambda)-2 f_{n-1}(\lambda)\right]$ is indeed the characteristic polynomial of the matrix

$$
A_{n+1}=\left(\begin{array}{ccccc}
0 & \sqrt{2} & 0 & \ldots & 0 \\
\sqrt{2} & 0 & 1 & \ldots & 0 \\
0 & 1 & 0 & \ldots & 0 \\
& \ldots & \ldots & & \\
0 & \ldots & & 0 & 1 \\
0 & 0 & \ldots & 1 & 0
\end{array}\right) .
$$

Notice that $f_{n}(\lambda)$ satisfies a type of Chebyshev polynomial equation, i.e.,

$$
f_{n}(x)=x f_{n-1}(x)-f_{n-2}(x) .
$$

The roots are $\cos [k \pi /(n+1)]$ where $k=1, \ldots, n$. Hence the eigenvalues of the matrix $A_{n+1}$ are $\cos [(2 k+1) \pi /(2 n+2)]$ where $k=0,1, \ldots, n$; among them, the largest is $\cos [\pi /(2 n+2)] \rightarrow 1$ as $n \rightarrow \infty$. So $w\left(S_{k}\right) \leq 1$ when $0 \leq k \leq \sqrt{2}$.

One may think that similar results will hold for bilateral weighted shifts, i.e., if $B_{k} \in B\left(\ell^{2}\right)$ is defined as $B_{k} e_{0}=k e_{1}$ and $B_{k} e_{n}=e_{n+1}$ for all nonzero integers $n$, then $W\left(B_{k}\right)$ will be the open unit disc for $0 \leq k \leq \sqrt{2}$. Indeed this is not true.

Proposition 3. If $B_{k} \in B\left(\ell^{2}\right)$ is defined as $B_{k} e_{0}=k e_{1}$ and $B_{k} e_{n}=e_{n+1}$ for $n \neq 0$, then

(i) $W\left(B_{k}\right)$ is the open unit disc if $0 \leq k \leq 1$, and

(ii) $w\left(B_{k}\right)>1$ if $k>1$.

Proof. (i) By Theorem 1 (ii), $W\left(B_{k}\right)$ is the open unit disc when $0 \leq k \leq 1$.

(ii) Assume that $k>1$. Notice that $W\left(B_{k}\right)$ is the bilateral weighted shift with weights $(\ldots, 1,1,(k), 1,1, \ldots)$. For $k>0, B_{k}$ is invertible and $B_{k}^{-1}$ is given as

$$
\begin{aligned}
& B_{k}^{-1} e_{n}=e_{n-1}, \quad n \neq 1, \\
& B_{k}^{-1} e_{1}=\frac{1}{k} e_{0},
\end{aligned}
$$

i.e, $B_{k}^{-1}$ shifts the $e_{n}$ 's backwards with the appropriate weights. Moreover, $B_{k}^{-1}$ $=R A_{k} R$ where $R e_{n}=e_{-n}$, for all integers $n$, is the reflection operator and $A_{k}$ is another bilateral weighted shift with weights $(\ldots, 1,1,(1), 1 / k, 1, \ldots)$. Since $R$ is unitary and $R^{*}=R, A_{k}$ and $B_{k}^{-1}$ are unitarily equivalent and we just consider $A_{k}$ 
instead of $B_{k}^{-1}$. It is known [H, Problem 85] that the spectra of $B_{k}$ and $B_{k}^{-1}$ are the unit circle. Concerning the numerical range, $W\left(B_{k}^{-1}\right)=W\left(A_{k}\right)$ is the open unit disc if $k>1$ by Theorem 1 (ii). By a result in [St, Cor. 1], if $w\left(B_{k}\right) \leq 1$, i.e., $W\left(B_{k}\right)$ is contained in the closed unit disc, then $B_{k}$ would be unitary. This is impossible except when $|k|=1$.

Neither the expression of $w\left(B_{k}\right)$ nor the closedness of $W\left(B_{k}\right)$ is known when $k>1$. However, since $W\left(S_{k}\right) \subset W\left(B_{k}\right)$, we have $w\left(B_{k}\right) \geq w\left(S_{k}\right)$, where $S_{k}$ is defined in Proposition 2.

Remark 2. In the finite dimensional case, $A$ is unitary if and only if the spectrum of $A$ lies on the unit circle and $w(A) \leq 1$. However, as pointed out in [CL], this is no longer valid for the infinite dimensional case. If $V$ is a Volterra operator, then $A=(I+V)^{-1}$ is such an example [H, Problem 150] since the spectrum of $A$ is $\{1\}$ and $\|A\|=1$. According to the proof of Proposition $3, B_{k}$ serves as another example for $k \neq 0,1$.

\section{Ridge's CONJECTURE}

In [R2, Theorem 1], it is proved that if $\left\{s_{n}\right\}$ is a periodic sequence of period $r$, then the numerical radius of the weighted shift is given by the expression $w(S)=$ $\max \left\{s_{1} x_{1} x_{2}+s_{2} x_{2} x_{3}+\cdots+s_{r} x_{r} x_{1}: x_{i} \in \mathbb{R}, x_{1}^{2}+\cdots+x_{r}^{2}=1\right\}$ and finding this is equivalent to solving a polynomial equation of degree $r$.

If $r=2$, the weights are $a, b, a, b, \ldots$ and $w(S)=(a+b) / 2, \rho(S)=\sqrt{a b}$ and $\|S\|=\max \{a, b\}$. If $r=3$, the weights are $a, b, c, a, b, c, \ldots$ and finding $w(S)$ leads to the cubic equation $\lambda^{3}-\left(a^{2}+b^{2}+c^{2}\right) \lambda-2 a b c=0$ [R2].

Ridge then [R2] surmised that for a weighted shift $S$ of periodic weights, $W(S)$ is open if and only if all the weights are nonzero. One implication is obvious: If some weight is zero, say, $s_{r}=0$, then $S$ is of the form $\oplus_{1}^{\infty} B$ where

$$
B=\left(\begin{array}{ccccc}
0 & 0 & 0 & \cdots & 0 \\
s_{1} & 0 & 0 & \cdots & 0 \\
0 & s_{2} & 0 & \cdots & 0 \\
\cdots & \cdots & \cdots & \cdots & \\
0 & \cdots & 0 & s_{r-2} & 0 \\
0 & 0 & \cdots & 0 & s_{r-1}
\end{array}\right)
$$

Then $W(S)=W(B)$ which is closed since $B$ is a finite dimensional operator. So we just consider the periodic weighted shift operators with positive weights.

Theorem 3. Suppose that $S \in B\left(\ell_{+}^{2}\right)$ is the weighted shift with positive periodic weights $\left\{s_{n}\right\}$ of period $r \geq 3$. Then the numerical radius, $w(S)$, is the Perron root of the nonegative irreducible symmetric matrix

$$
S^{\prime}=\frac{1}{2}\left(\begin{array}{cccccc}
0 & s_{1} & 0 & 0 & \ldots & s_{r} \\
s_{1} & 0 & s_{2} & 0 & \ldots & 0 \\
0 & s_{2} & 0 & s_{3} & \ldots & 0 \\
\ldots & \ldots & \ldots & \ldots & \ldots & \\
0 & \ldots & 0 & s_{r-2} & 0 & s_{r-1} \\
s_{r} & 0 & \ldots & 0 & s_{r-1} & 0
\end{array}\right)
$$

Proof. Indeed, it is obvious $w\left(S^{\prime}\right)=\max \left\{s_{1} x_{1} x_{2}+s_{2} x_{2} x_{3}+\cdots+s_{r} x_{r} x_{1}: x_{i} \geq\right.$ $\left.0, x_{1}^{2}+\cdots+x_{r}^{2}=1\right\}=w(S)$ by $\left[\mathrm{R} 2\right.$, Theorem 1]. Since $S^{\prime}$ is a nonnegative 
irreducible symmetric matrix, $W\left(S^{\prime}\right)$ is a closed interval on $\mathbb{R}$ and hence $w\left(S^{\prime}\right)$ is the the largest eigenvalue, in absolute value, of $S^{\prime}$. This is the Perron root of $S^{\prime}$.

When $r=3$ and $S$ is periodic weighted shift operator with weights $a, b, c$, the characteristic equation of

$$
S^{\prime}=\frac{1}{2}\left(\begin{array}{lll}
0 & a & c \\
a & 0 & b \\
c & b & 0
\end{array}\right)
$$

is the cubic equation $\lambda^{3}-\left(a^{2}+b^{2}+c^{2}\right) \lambda-2 a b c=0$. So the numerical radius of $S$ is the largest root of the cubic equation, i.e., the largest of

$$
A+B, \quad \frac{-A+B}{2}+\frac{A-B}{2} \sqrt{3}, \quad \frac{-A+B}{2}-\frac{A-B}{2} \sqrt{3}
$$

where

$$
\begin{aligned}
& A=\sqrt[3]{a b c+\sqrt{a^{2} b^{2} c^{2}-\frac{\left(a^{2}+b^{2}+c^{2}\right)^{3}}{27}}}, \\
& B=-\sqrt[3]{-a b c+\sqrt{a^{2} b^{2} c^{2}-\frac{\left(a^{2}+b^{2}+c^{2}\right)^{3}}{27}}} .
\end{aligned}
$$

One may notice that the numerical radius of $S$ is independent of the relative positions of $a, b, c$ as well as the $r=2$ case. However this is no longer valid when $r=4$.

Example 3. If

$$
A=\left(\begin{array}{llll}
0 & 1 & 0 & 4 \\
1 & 0 & 2 & 0 \\
0 & 2 & 0 & 3 \\
4 & 0 & 3 & 0
\end{array}\right), \quad B=\left(\begin{array}{llll}
0 & 4 & 0 & 1 \\
4 & 0 & 2 & 0 \\
0 & 2 & 0 & 3 \\
1 & 0 & 3 & 0
\end{array}\right)
$$

then the Perron roots of $A$ and $B$ are approximately 5.3983 and 5.1167, respectively. These are the numerical radii of the periodic weighted shifts with weights $1,2,3,4$ and $4,2,3,1$, respectively. Indeed if $r=4$ and the weights are $a, b, c, d$, then the characteristic equation of

$$
S^{\prime}=\frac{1}{2}\left(\begin{array}{cccc}
0 & a & 0 & d \\
a & 0 & b & 0 \\
0 & b & 0 & c \\
d & 0 & c & 0
\end{array}\right)
$$

is $\lambda^{4}-\lambda^{2}\left(a^{2}+b^{2}+c^{2}+d^{2}\right)-2 a b c d+d^{2} b^{2}+a^{2} c^{2}=0$. Hence the largest root of the equation is

$$
\begin{aligned}
& \sqrt{\frac{\left(a^{2}+b^{2}+c^{2}+d^{2}\right)+\sqrt{\left(a^{2}+b^{2}+c^{2}+d^{2}\right)^{2}-4\left(-2 a b c d+d^{2} b^{2}+a^{2} c^{2}\right)}}{2}} \\
& =\sqrt{\frac{\left(a^{2}+b^{2}+c^{2}+d^{2}\right)+\sqrt{\left(a^{2}+b^{2}-c^{2}-d^{2}\right)^{2}+8 a b c d+4 a^{2} d^{2}+4 b^{2} c^{2}}}{2}} .
\end{aligned}
$$


Example 4. If

$$
A=\left(\begin{array}{lllll}
0 & 1 & 0 & 0 & 5 \\
1 & 0 & 2 & 0 & 0 \\
0 & 2 & 0 & 3 & 0 \\
0 & 0 & 3 & 0 & 4 \\
5 & 0 & 0 & 4 & 0
\end{array}\right), \quad B=\left(\begin{array}{lllll}
0 & 5 & 0 & 0 & 1 \\
5 & 0 & 2 & 0 & 0 \\
0 & 2 & 0 & 3 & 0 \\
0 & 0 & 3 & 0 & 4 \\
1 & 0 & 0 & 4 & 0
\end{array}\right)
$$

then the Perron roots of $A$ and $B$ are approximately 6.8501 and 6.0972 , respectively. These are the numerical radii of the periodic weighted shifts with weights $1,2,3,4,5$ and 5,2,3,4,1 respectively. So, in general, the numerical radius of the shift with periodic weights depends on the relative positions of the weights.

The following result resolves the conjecture of Ridge.

Theorem 4. If $S \in B\left(\ell_{+}^{2}\right)\left(B \in B\left(\ell^{2}\right)\right)$ is the unilateral (bilateral) weighted shift with nonzero periodic weights, then $W(S)(W(B))$ is an open disc centered at the origin.

Proof. Unilateral case. Case 1. $r=2$. Let $a, b>0$ be the weights. By [R2], $(a+b) / 2$ is the numerical radius of $S$. It is sufficient to show that $(a+b) / 2$ is not an eigenvalue of $S_{+}$in view of Corollary 3. Suppose on the contrary that there were a nonzero $x \in \ell_{+}^{2}$ such that $S_{+} x=\frac{a+b}{2} x$. Then we would have

$$
\begin{aligned}
a x_{2} & =(a+b) x_{1}, \\
a x_{2 n-1}+b x_{2 n+1} & =(a+b) x_{2 n}, \quad n=1,2, \ldots, \\
b x_{2 n}+a x_{2 n+2} & =(a+b) x_{2 n+1}, \quad n=1,2, \ldots
\end{aligned}
$$

Clearly $x_{1} \neq 0$, for otherwise $x=0$. Adding the last two equations yields

$$
a\left(x_{2 n-1}+x_{2 n+2}\right)=a\left(x_{2 n}+x_{2 n+1}\right),
$$

i.e., $x_{2 n+2}-x_{2 n}=x_{2 n+1}-x_{2 n-1}, n=1,2, \ldots$. Similarly we have $x_{2 n+1}-x_{2 n-1}=$ $x_{2 n}-x_{2 n-2}, n=1,2, \ldots$ with $x_{0}=0$. So the two subsequences $\left\{x_{2 n}\right\}$ and $\left\{x_{2 n+1}\right\}$ are in arithmetic progression with common difference $d$, i.e., $x_{2 n}=x_{2}+(n-1) d$ and $x_{2 n+1}=x_{1}+n d$. But $x \in \ell_{+}^{2}$. So $x_{k} \rightarrow 0$ as $k \rightarrow \infty$ and hence contradicts the arithmetic progression of $\left\{x_{2 n+1}\right\}$. Hence $(a+b) / 2$ is not an eigenvalue of $S_{+}$.

Case 2. $r \geq 3$. Similarly it is sufficient to show that $\lambda$, the Perron root of $S^{\prime}$, is not an eigenvalue of the operator $S_{+}$. Suppose on the contrary that there were a nonzero $x \in \ell_{+}^{2}$ such that $S_{+} x=\lambda x$. Notice that $x_{1} \neq 0$, otherwise $x=0$. We can pick $x_{1}>0$.

We claim that $x>0$. The reason is that according to Theorem 3 , we have

$$
w(S)=\lambda=\frac{(S x, x)}{(x, x)}=\frac{s_{1} x_{1} x_{2}+s_{2} x_{2} x_{3}+\ldots}{(x, x)} .
$$


By the triangle inequality,

$$
\begin{aligned}
w(S) & =\frac{s_{1} x_{1} x_{2}+s_{2} x_{2} x_{3}+\ldots}{(x, x)} \\
& =\frac{\left|s_{1} x_{1} x_{2}+s_{2} x_{2} x_{3}+\ldots\right|}{(x, x)} \\
& \leq \frac{s_{1}\left|x_{1}\right|\left|x_{2}\right|+s_{2}\left|x_{2}\right|\left|x_{3}\right|+\ldots}{(x, x)} \\
& =\frac{(S|x|,|x|)}{(|x|,|x|)} \in W(S)
\end{aligned}
$$

where $|x|=\left(\left|x_{1}\right|,\left|x_{2}\right|, \ldots\right)^{T}$ and $x$ have the same norm. Hence we have $x \geq 0$.

If some $x_{j}=0$ then let $k \geq 2$ be the first index such that $x_{k}=0$. So $S^{\prime} x=\lambda x$ would provide

$$
a x_{k-1}+b x_{k+1}=2 \lambda x_{k},
$$

where $a$ and $b$ are consecutive $s$ 's (maybe $s_{r}$ and $s_{1}$ ). This implies that $x_{k-1} x_{k+1}<$ 0 which is impossible.

Now consider the following system of infinitely many equations:

$$
\begin{aligned}
s_{r} y_{0}+s_{1} y_{2} & =2 \lambda y_{1}, \\
s_{1} y_{1}+s_{2} y_{3} & =2 \lambda y_{2}, \\
\vdots & \\
s_{r-1} y_{r-1}+s_{r} y_{r+1} & =2 \lambda y_{r}, \\
s_{r} y_{r}+s_{1} y_{r+2} & =2 \lambda y_{r+1}, \\
s_{1} y_{r+1}+s_{2} y_{r+3} & =2 \lambda y_{r+2}, \\
\vdots & \\
s_{r-1} y_{2 r-1}+s_{r} y_{2 r+1} & =2 \lambda y_{2 r},
\end{aligned}
$$

The equation $S_{+} x=\lambda x$ means that the vector $\left(x_{0}, x_{1}, x_{2}, \ldots\right)^{T}$ is a solution to the system where $x_{0}=0$ and we can set $x_{1}=1$. Notice that the solution set is a linear space because everything is linear. The dimension of the solution set is 2 as $y_{0}$ and $y_{1}$ will determine other $y$ 's. Moreover $\left(x_{r}, x_{r+1}, x_{r+2}, \ldots\right)^{T}$ is also a solution to the system due to the recursive pattern. Furthermore, if $z=\left(z_{1}, z_{2}, \ldots z_{r}\right)^{T}>0$ is the Perron vector of the matrix $S^{\prime}$, i.e., $S^{\prime} z=\lambda z$, then the vector

$$
\left(z_{r}, z_{1}, z_{2}, \ldots, z_{r-1}, z_{r}, z_{1}, z_{2}, \ldots, z_{r-1}, \ldots\right)^{T}
$$

is also a solution of the same system. This is because that $S^{\prime} z=\lambda z$ is equivalent to

$$
\begin{aligned}
s_{r} z_{r}+s_{1} z_{2} & =2 \lambda z_{1}, \\
s_{1} z_{1}+s_{2} z_{3} & =2 \lambda z_{2}, \\
\vdots & \\
s_{r-1} z_{r-1}+s_{r} z_{1} & =2 \lambda z_{r} .
\end{aligned}
$$


So the three vectors

$\left(x_{0}, x_{1}, x_{2}, \ldots\right)^{T},\left(x_{r}, x_{r+1}, x_{r+2}, \ldots\right)^{T},\left(z_{r}, z_{1}, z_{2}, \ldots, z_{r-1}, z_{r}, z_{1}, z_{2}, \ldots, z_{r-1}, \ldots\right)^{T}$

are linearly dependent. The first two vectors are linearly independent since $x_{0}=0$ and $x_{r}>0$. So there exist scalars $\alpha$ and $\beta$ such that

$$
\begin{aligned}
\left(z_{r}, z_{1}, z_{2}, \ldots, z_{r-1}, z_{r}, z_{1}, z_{2}, \ldots, z_{r-1}, \ldots\right)^{T} & \\
= & \alpha\left(x_{0}, x_{1}, x_{2}, \ldots\right)^{T}+\beta\left(x_{r}, x_{r+1}, x_{r+2}, \ldots\right)^{T} .
\end{aligned}
$$

This implies that $z_{r}=\alpha x_{k r}+\beta x_{(k+1) r}$ for any nonnegative integer $k$. However, $x \in \ell_{+}^{2}$ and hence $x_{n} \rightarrow 0$ as $n \rightarrow \infty$. As a result $z_{r}=0$ which is a impossible.

\section{Bilateral case.}

Case 1. $r=2$. The argument for the unilateral case works for the bilateral case.

Case 2. $r \geq 3$. We try to show that the Perron root of $S^{\prime}, \lambda$, is not an eigenvalue of the operator $B_{+}$. Suppose on the contrary that there were a nonzero

$$
x=\left(\ldots, x_{-2}, x_{-1},\left(x_{0}\right), x_{1}, x_{2}, \ldots\right)^{T} \in \ell^{2},
$$

such that $B_{+} x=\lambda x$. Now consider the following system of infinitely many equations:

$$
\begin{aligned}
& \\
s_{r} y_{-r}+s_{1} y_{-r+2} & =2 \lambda y_{-r+1}, \\
s_{1} y_{-r+1}+s_{2} y_{-r+3}= & 2 \lambda y_{-r+2}, \\
\vdots & \\
s_{r-1} y_{-1}+s_{r} y_{1}= & 2 \lambda y_{0}, \\
s_{r} y_{0}+s_{1} y_{2}= & 2 \lambda y_{1}, \\
s_{1} y_{1}+s_{2} y_{3}= & 2 \lambda y_{2}, \\
\vdots & \\
s_{r-1} y_{r-1}+s_{r} y_{r+1} & =2 \lambda y_{r}, \\
s_{r} y_{r}+s_{1} y_{r+2} & =2 \lambda y_{r+1}, \\
s_{1} y_{r+1}+s_{2} y_{r+3} & =2 \lambda y_{r+2}, \\
\vdots & \\
s_{r-1} y_{2 r-1}+s_{r} y_{2 r+1} & =2 \lambda y_{2 r},
\end{aligned}
$$

Again the solution set is a two dimensional vector space because everything is linear and $y_{0}$ and $y_{1}$ will determine other $y$ 's. Then $x=\left(\ldots, x_{-2}, x_{-1},\left(x_{0}\right), x_{1}, x_{2}, \ldots\right)^{T}$ is a solution. Moreover, due to the recursive relation, the vectors

$$
x^{(k)}=\left(\ldots, x_{k r-2}, x_{k r-1},\left(x_{k r}\right), x_{k r+1}, x_{k r+2}, \ldots\right)^{T}, \quad k=1,2, \ldots,
$$

are solutions. Indeed $x^{(k)}$ is obtained from $x$ by shifting the vector $x$ towards the right side by $k r$ slots, i.e., $x^{(k)}=V^{k r} x$ if $V$ is the usual bilateral shift. 
We claim that one of the pairs of $\left\{x, x^{(k)}\right\}, k=1,2 \ldots$, must be a linearly dependent set. Otherwise, there exists a nonzero $\alpha_{k}$ such that $x=\alpha_{k} x^{(k)}$ where $k=1,2, \ldots$. Obviously $\|x\|=\left\|x^{(k)}\right\|$ as $V$ is a unitary operator. So we have $\left|\alpha_{k}\right|=1$ for all positive integers $k$. It means that $\left|x_{-k r}\right|=\left|x_{0}\right|$ and $\left|x_{-k r+1}\right|=\left|x_{1}\right|$ for all positive integers $k$. Notice that $x_{0}$ and $x_{1}$ cannot be both zeros (otherwise $x=0)$. Then it implies that $x \notin \ell^{2}$ as $\lim _{n \rightarrow-\infty} x_{n} \neq 0$.

Let $x, x^{(j)}$ be linearly independent vectors for some $j$. Then we consider the Perron vector of $S^{\prime}, z>0$, as we did in the unilateral case, i.e., $S^{\prime} z=\lambda z$. The positive vector

$$
u=\left(z_{r}, z_{1}, z_{2}, \ldots, z_{r-1}, z_{r}, z_{1}, z_{2}, \ldots, z_{r-1}, \ldots\right)^{T}
$$

is also a solution. So there exist scalars $\alpha$ and $\beta$ such that

$$
u=\alpha x+\beta x^{(j)} \text {. }
$$

But $\lim _{n \rightarrow \infty} x_{n}=0$. It leads to a contradiction.

Remark 3. Indeed $\left\{x_{n}\right\}$ is a strictly increasing sequence for the unilateral weighted shift when $r=2$. The proof goes as follows: Solving the same equations, we have

$$
\begin{aligned}
x_{2} & =\frac{(a+b)}{a} x_{1}, \\
x_{2 n+1} & =\frac{1}{b}\left[(a+b) x_{2 n}-a x_{2 n-1}\right], \\
x_{2 n+2} & =\frac{1}{a}\left[(a+b) x_{2 n+1}-b x_{2 n}\right] .
\end{aligned}
$$

Then $x_{2}>x_{1}$. Moreover,

$$
x_{2 n+1}=x_{2 n}+\frac{a}{b}\left(x_{2 n}-x_{2 n-1}\right)>x_{2 n},
$$

and

$$
x_{2 n+2}=x_{2 n+1}+\frac{a}{b}\left(x_{2 n+1}-x_{2 n}\right)>x_{2 n+1},
$$

by induction, i.e., $\left\{x_{n}\right\}$ is a strictly increasing sequence.

With respect to the above argument, one may be tempted to assume that if the Perron root, $\lambda$, of the symmetric nonnegative irreducible matrix $S^{\prime}$ is greater than or equal to $\frac{1}{2} \max \left\{s_{1}+s_{2}, s_{2}+s_{3}, \ldots s_{r-1}+s_{r}, s_{r}+s_{1}\right\}$, then $W(S)$ is open. But the imposed assumption implies that [HJ, p.356] every Gersgorin circle passes through $\lambda$ (i.e., (i) if $r$ is an odd integer, then $s_{n}, n=1, \ldots r$, are identical, i.e., $r=1$; (ii) if $r$ is an even integer, then $s_{2 n}=a$ and $s_{2 n+1}=b$, i.e., $r=2$ ). They were already treated in Theorem 1 and Theorem 4 respectively.

The following example shows that the sequence $\left\{x_{n}\right\}$ is not necessarily increasing when $r \geq 3$.

Example 5. Let $S$ be the weighted shift with periodic weights 1,2,3. Set $x_{1}=1$. We list the first eight terms of the sequence $\left\{x_{n}\right\}$ :

$$
\{1.0000,4.1131,7.9588,8.1696,9.7262,15.9175,15.3393,15.3393, \ldots\} \text {. }
$$

The author would like to express thanks to Peter Nylen pointing out the reference in $[\mathrm{HJ}]$. 


\section{REFERENCES}

[BS] C. A. Berger and J. G. Stampfli, Mapping theorems for the numerical range, Amer. J. Math. 89 (1967), 1047-1055. MR 36:5744

[C] J. B. Conway, The Theory of Subnormal Operators, Math. Surveys and Monographs, Vol. 36, Amer. Math. Soc., Providence, R.I., 1991. MR 92h:47026

[CL] M. D. Choi and C.K. Li, Numerical ranges of the powers of an operator, preprint.

[Ch] T. R. Chow, The spectral radius of a direct integral of operators, Math. Ann. 188 (1970), 285-303.

[H] P. Halmos, A Hilbert Space Problem Book, Springer-Verlag, New York, 1978. MR 84e:47001

[HJ] R. A. Horn and C. R. Johnson, Topics in Matrix Analysis, Cambridge Univ. Press, 1990. MR 92e:15003, MR 95c: 15001

[L] B.V. Limaye, Functional Analysis, Wiley, New Delhi, 1981. MR 83b:46001

[MS] M. Marcus and B. N. Shure, The numerical range of certain 0,1-matrices, Linear and Multilinear Algebra, 7 (1979), 111-120. MR 80c:15015

[R1] W. Ridge, Approximate point spectrum of a weighted shift, Trans. Amer. Math. Soc., 147 (1970), 349-356. MR 40:7843

[R2] W. Ridge, Numerical range of a weighted shift with periodic weights, Proc. Amer. Math. Soc., 55 (1976), no. 1, 107-110. MR 53:1332

[S] A. L. Shields, Weighted shift operators and analytic function theory, Math. Surveys, no. 13, Amer. Math. Soc., Providence, R.I., 1974, p.49-128. MR 50:14341

[St] J. G. Stampfli, Minimal range theorem for operators with thin spectra, Pac. J. Math., 23 (1967), 601-612. MR 37:4655

Department of Mathematics, Auburn University, Alabama 36849-5310

E-mail address: tamtiny@mail.auburn.edu 